\title{
An Evaluation Synthesis of US AIDS Drug Assistance Program Policy
}

\author{
Michael A. Horneffer, Y. Tony Yang*
}

Department of Health Administration and Policy, George Mason University, Fairfax, USA.

Email: *ytyang@gmu.edu

Received February $15^{\text {th }}, 2013$; revised March $17^{\text {th }}, 2013$; accepted March $26^{\text {th }}, 2013$

Copyright @ 2013 Michael A. Horneffer, Y. Tony Yang. This is an open access article distributed under the Creative Commons Attribution License, which permits unrestricted use, distribution, and reproduction in any medium, provided the original work is properly cited.

\begin{abstract}
US Congress passed the CARE Act in 1990 in response to a dramatically growing need for resources to combat the AIDS epidemic. One of the programs contained in the Act was the AIDS Drug Assistance Program (ADAP), a federally-funded but state-maintained and managed program primarily concerned with providing medication for low-income HIV/AIDS patients. While ADAP programs across the country reached one-third of all patients in 2007, these programs are now in budgetary danger due to the economic recession, state budgetary constraints, the rising cost of healthcare generally, and longer life expectancies associated with current highly active antiretroviral therapy (HAART). This paper first evaluates the current state of ADAP, its strengths and weaknesses, and examines its sustainability in the short term if short-term measures are taken. Concluding that such measures would not lead to long-term sustainability, this paper then argues for a long-term solution to ADAP's current problems, namely a national, centralized ADAP standard for budgetary and administrative matters. Such a program would increase the long-term sustainability and effectiveness of current ADAP programs by employing more efficient, standard policies and allowing larger, wholesale purchases of costly HAART medications. Moreover, a national policy would address the disparity that currently exists in ADAP programs today with regard to both minorities and those on the waiting lists for treatment. The institution of a national ADAP program would certainly face many political hurdles. Consequently, this paper also looks to a recent political dispute, the enactment of the Affordable Care Act (ACA), for guidance. Using the passage of the ACA as an example could light the path for passage of a national ADAP standard. Ultimately, this would lead to a more effective and sustainable program for HIV/AIDS patients in the United States.
\end{abstract}

Keywords: HIV; AIDS; Drug Policy; Program Evaluation; Health Reform

\section{Introduction}

HIV/AIDS continues to be considered an epidemic by public health professionals and physicians. Despite the advent of new drugs ("HAART," or "highly active antiretroviral therapy") and prevention programs, HIV/AIDS is still very much prevalent in the United States. While the prevention and treatment of HIV has been made more manageable due to new drugs, these medications are often inaccessible for patients because of their high costs. This paper evaluates the national ADAP (AIDS Drug Assistance Programs) initiative. Whether ADAP is effective in reaching its intended recipients, the future sustainability of the program, possible solutions to ADAP budgetary shortcomings, as well as the effect of a new

\footnotetext{
"Corresponding author.
}

national health policy on ADAP will be discussed and incorporated into an overall assessment of the program.

\section{ADAP Overview}

On August 18, 1990, in response to the drastic need for resources to combat the HIV/AIDS epidemic, Congress passed the CARE Act. In 2009, the CARE Act was reenacted and renamed the "Ryan White HIV/AIDS Treatment Extension Act" [1]. The Act is divided into five parts (A, B, C, D, F) and supplements the resources of states and municipalities in combating HIV/AIDS. It provides financial assistance in the areas of primary care, general HIV/AIDS services delivery, health care for women, infants and children with HIV/AIDS, AIDS education and training, as well as medication for HIV/ AIDS. Under Part B of the Ryan White HIV/AIDS 
Treatment Extension Act, the AIDS Drugs Assistance Program was developed as a "federally funded, statebased program [to] provid[e] medications to uninsured HIV-infected persons, or those with limited resources” [2].

HIV/AIDS was originally treated by nucleoside analog reverse-transcriptase inhibitors or NRTI's [3]. NRTI's, while being the first successful treatment regimens developed to combat HIV/AIDS, were not shown to be solely effective for prolonged and successful treatment of the virus. In the mid 1990’s, nonnucleoside reverse transcriptase inhibitors (NNRTIs) and protease inhibitors (PI's) were developed [2]. NNRTIs and PI's revolutionized HIV/ AIDS care. Used in highly active anti-retroviral therapy (HAART), these new classes of drugs extended the life expectancy of HIV positive individuals by increasing treatment efficacy and reducing side-effects related to drug toxicity [4]. These new drugs come at a cost, however. Treatment with HAART can range from \$10,000 $\$ 12,000$ per person annually” [2]. ADAPs were created to help offset the high costs associated with the HAART regimens, especially for low-income patients.

With more than " 183,000 enrollees in FY 2007... [ADAPs] reached over a third of all people with HIV receiving care in the United States" [5]. The program is funded by both the state and federal governments. The federal "ADAP earmark, one of the four main ADAP funding streams and designated specifically for ADAP by Congress each year, is the largest component of the budget [at 51\%, or 774 million in FY 2008]" [5]. Despite being the largest component of ADAP, federal funding no longer drives the program's budgetary growth. Instead, other funding streams such as, "drug rebates, and state general revenue support," which vary and are subject to contractual arrangements and budgetary allocations, are now the primary drivers of growth [5].

Because an expansion of testing programs has led to an increase in awareness of HIV/AIDS contraction, the number of new cases of HIV/AIDS infection is steadily rising [5]. The national economic recession has also placed an additional burden on ADAP programs as more individuals, having lost their insurance benefits due to unemployment, begin to rely on the program for medications. Government responses to the recession, such as state budgetary restraints and cutbacks, also adversely impact resources available to ADAP programs. With increasing numbers of under or uninsured persons with HIV and diminishing state and federal resources, ADAPs face significant challenges.

\section{Effectiveness}

In addressing the effectiveness of ADAP, one must first examine the costs and benefits of maintaining ADAP funding in light of the current and future fiscal challenges associated with the program. How ADAP is structured and administered must also be incorporated into a study of its effectiveness. Expanding and continuing coverage of ADAP is expensive due to the rising cost of medications, which also prolong survival. In fact, it is estimated that from the time of entering care with HAART, the average HIV-positive patient's life expectancy is 24.2 years with an average cost of $\$ 618,900$ per person for all medical care [4]. While the average program beneficiary's life expectancy has increased with the advent of HAART, costs associated with the new treatment regimen are distributed over a much longer time span. Once on HAART, an individual must continue to receive the medications for the rest of his or her life.

Moving the bulk of HIV positive individuals from an acute to chronic health state by expanding ADAP enrollment, defrays short term costs [2]. People who are constantly hospitalized or are too sick cannot work. If ADAP coverage were to be extended, "patients [would be more healthy and thus] more likely to work" [3]. It has also been shown that being able to work increases the likelihood of finding private insurance. ADAP, however, is used only to cover the under or uninsured. In a comparison study, which projected costs of offering more "generous ADAP “ versus "less generous ADAP," it was found that "better access to [ADAP would result in] decreased costs [by] a statistically and economically significant 30 percent" [3]. This associated cost decrease is primarily related to the decrease in costly hospitalizations. Another benefit of HAART utilization is that the medications decrease the likelihood of having to use other prescription drugs to treat the opportunistic infections that develop due to the onset of acute HIV or AIDS [3].

In examining the cost of ADAP over the long run, however, the savings associated with lower inpatient care costs due to HAART are offset by the high costs of the medications and by increasing demand for ADAPs [6]. Between 1999 and 2000 (pre-recession), there was a 12\% increase in the number of ADAP clients, as well as a $22 \%$ increase in expenses related to HAART [6]. As the recession has already resulted in an increase in the number of HIV positive enrollees due to lack of health insurance from unemployment, further increasing the number enrollees would only serve to further burden the already overextended ADAPs. Most studies on the fiscal benefit of increasing ADAP enrollment in the short term fail to account for the long-term fiscal implications of maintaining and increasing the program. HAART is more successful at preventing the progression from HIV to AIDS over a longer period of time than previous treatment regimens. Thus, expanding program coverage will only result in a greater number of ADAP program beneficiaries who will require extended HAART treatment 
[1]. These beneficiaries will live longer because of the effectiveness of HAART, but at the same time "incur [greater] costs over more years due to improved life expectancies" [4]. For these reasons, the necessary programmatic adjustments required to increase ADAP enrollment, while beneficial in the short term, are not realistically implementable in the long term, mainly due to state budgetary restraints.

While the Federal government remains the largest funder of ADAP, program growth is now primarily driven by the States [5]. States are also the primary agents in the administration of ADAP [3]. Since the recession of 2007, however, state contribution and matching funds to ADAP have decreased from $\$ 273,610,088$ ('07 total) to $\$ 248,708,459$ ('08 total) and \$4,648,077 ('07 total) to $\$ 2,564,830$ ('08 total), respectively [1]. In order to meet budgetary constraints, states have also "restrict[ed] the number of enrollees, constrain[ed] coverage to specific classes of drugs, and impose[d] spending limits on beneficiaries" [3]. Coverage also often depends on the state definition of poverty, with minimal income eligibility ranging from " $125 \%$ of the federal poverty level... to $500 \%$ of the federal poverty level" [6]. It has also become a common practice for states to adjust their minimal income eligibility ranges in order to accommodate fewer public program beneficiaries and thusly save money.

State variations in ADAP administration and coverage result in unequal treatment of program enrollees. These variations depend upon the resources available to the state in which the enrollee resides [6]. Various mathematical models which evaluated state-to-state differences in ADAP policies demonstrated that these differences "could produce [a] variation of up to 4.25 years in lifeexpectancy estimates for clinically similar patients" [6]. The absence of a national ADAP formulary standard results in unequal treatment of program enrollees. Thus, ADAP is by no means effective in achieving an equitable distribution of its resources.

\section{ADAP and Minority Groups}

Many academics and policy makers assert that those traditionally underserved by ADAP, yet most affected by the HIV/AIDS epidemic, are minorities—particularly African Americans. Whether scarce resources should be allocated on the basis of race must be assessed. In a comparison study between the four states with the largest HIV/AIDS population (New York, California, Florida, and Texas), "African Americans represented a higher proportion of people living with AIDS than their proportion in the overall population [7]. The same study also indicated that African Americans were "less likely to receive protease-inhibitors than non-Latino whites" [7].

While this study identified social barriers (e.g., distrust of government and traditional medicine, lack of informa- tion, HIV-related stigma) associated with different ethnic groups as possible explanations for this trend, policy and structural issues of state-administered ADAP programs seemed to be most salient, given that "[a]ccess to HIV pharmaceuticals depends greatly on the state in which an individual lives" [7]. Rather than re-tailoring each state's individual ADAP policy towards disadvantaged groups, a nationally standardized policy and eligibility criterion would systematically address the issue across all states. This national ADAP standard would eliminate barriers of access for low income individuals. These barriers have a disproportionate effect on members of racial/ethnic minority groups [7]. However, the creation of a new national ADAP policy might also result in a greater fiscal strain on poorer states [8].

It should be recognized that assertions that African Americans are underserved by ADAPs fail to take into account other avenues by which these minorities may receive HAART. As African Americans are more likely than whites to have public or no health insurance, they are more likely to be receiving care through Medicaid [9]. Despite rapidly diminishing ADAP-related funding and resources, the poor and uninsured, who are more likely to be African American, may still receive comparable HAART medication subsidies through Medicaid. Thus, "it may be unnecessary for [those] individuals requiring pharmaceuticals to use ADAP” [9].

In another analysis of New York, California, Florida, and Texas, "a total of 154,196 HIV-infected individuals enrolled in... state programs [were examined]" [9]. The study revealed that across the four states, Medicaid programs had larger numbers of enrollees than ADAP [9]. In addition, African Americans participation in Medicaid programs exceeded their representation among people living with AIDS [9]. This indicates that while HIVpositive African-Americans might not be among the majority of enrollees in ADAP, they constitute the largest Medicaid supported HIV-positive population. The only exception to this dynamic was exhibited in California in which the majority of new cases and people living with HIV/AIDS were non-Latino whites [9]. These HIVpositive African Americans in California also "participated in [the state] Medicaid [program] above their representation among people living with AIDS” [9].

In all four states, African Americans were the largest represented racial group among HIV-positive Medicaid recipients. Lower percentages of representation in the ADAP category does not necessarily mean "under-served." As African Americans are more likely to meet the eligibility criterion of Medicaid, they are more likely to enroll in that program [9]. Furthermore, African Americans comprise the second largest racial/ethnic group reached by ADAP at 33\%. Non-Hispanic Whites comprise the largest group reached by ADAP at 39\% [1]. 
More relevant studies might instead examine the racial/ ethnic groups that comprise the largest percentage of new HIV/AIDS infections, rather than comparing the numbers of current ADAP enrollees by race.

\section{Sustainability}

With increasingly diminished financial resources available to ADAPs, any policy aimed at increasing, or even maintaining, enrollment can only mean longer waiting lists, more medication formulary reductions, or other measures enacted to reduce costs and, more often than not, reduce coverage. This trend has already been observed in many states. Thus, currently, "fewer clients are served in ADAPs than are enrolled at any given time" [5]. Some states have enacted waiting lists in response to this, which often require patients to employ stopgap, time consuming measures to obtain drugs while waiting to obtain full cost coverage from the state ADAP [5]. Others have narrowed eligibility criteria or reduced the classes of drugs covered by the state ADAP [5].

At the same time that ADAPs are under-funded and forcing states to take cost-cutting measures, the projected lifetime total costs of HIV medical care is $\$ 618,900$ per person. If rationing policies and rebate and wholesale purchasing schemes for HAART medications are implemented (models that will be discussed in a later section), this cost can be reduced to $\$ 385,200$ per person [4]. Based on this, it follows that the bulk of long-term treatment expenses for HIV/AIDS has to do with the HAART medications. HAART itself, however, has negative implications on the long-term sustainability of ADAP because of its effectiveness.

Long-term life expectancy will most likely increase with the continued use of HAART. According to one cost projection mode, this increase in life expectancy, however, will also incur "total costs [ranging] from 39\% to 91\% higher" than previous non-HAART treatment regimens [2]. This model differs from most observational studies, "which report decreases in total HIV care costs" [2]. The difference can be attributed to the short, i.e., less than 5-year, time horizons of those studies, which fail to account for the fact that the longer life expectancy associated with HAART means providing HAART to patients for a longer periods of time [2]. Future studies should focus on lifetime costs of treatment, as well as complications arising from viral resistance, long-term complication associated with HIV/AIDS care, as well as the potential impact of newer therapeutic options.

What can be extrapolated from the foregoing discussion is that the long-term sustainability of many ADAP programs is in jeopardy. Federal funding is stagnant and state budgets are already overburdened from economic recession and high levels of debt. Moreover, the current favored treatment for HIV/AIDS will, in the long run, lead to more strain on state ADAPs. Consequently, the long term viability of ADAPs should be of serious concern to health policy makers. On one hand, while more restrictive ADAP programs may not be optimal medical policy, it would allow for continued coverage of large numbers of people. As the number of people relying on ADAP increases due to lack of adequate insurance and the rising number of new HIV/AIDS infections, scarce resources must be allocated according to greatest need. On the other hand, it should be noted that while rationed programs "incur lower medication costs and lower total costs for the system... [they] are [also] associated with lower life expectancy" [2]. Thus, there are also certainly ethical dilemmas underlying policies that restrict access to ADAPs as well.

\section{Solutions to the ADAP Crisis}

There is no uniform, i.e., national, ADAP policy formula. States determine how much funding is allocated to their ADAPs, as well as how their program's limited resources are distributed. Some states take new program enrollees on a "first come, first serve basis," while other states consider medical need [10]. Legislators intended for ADAP to "provide... medications to low income individuals living with HIV... who have little or no coverage from private or third party insurance" [1]. As ADAP is a medical program, resources should be distributed according to greatest medical need. Enrolling new clients on the basis of a restrictive medical criterion, rather than the creation of waitlists, would better allocate scarce resources as well as more fairly distribute program resources.

In light of ADAP waiting lists and an ever more restrictive budgetary climate, a new ADAP standard which accepts new enrollees on the basis of CD4 cell count "would serve more-diverse patient populations with significantly lower CD4 cell counts than would a first-come, first served approach" [10]. HIV infected individuals with a lower CD4 cell counts are more susceptible to opportunistic infections and illnesses, which may require expensive hospitalization. Current CDC guidelines state that HIV-positive individuals should commence antiretroviral therapy "at a CD4 cell count of 200 - 350 cells/ $\mu L$ " [10]. A model-based study simulated the effect of a restrictive ADAP policy which accepted only clients with a CD4 count equal to or less than 350 cells/ $\mu \mathrm{L}$ [10]. All ADAP data pertaining to rates of new enrollees used in this study were taken from the state of Massachusetts. Despite the methodological limitations associated with using a single state's dataset to base a standard formulary policy, the model could be regenerated using ADAP data from any state. Adverse budgetary conditions were also simulated in order to examine the impact of the new CD4 restrictive client criterion in a particularly unfavorable 
ADAP budgetary climate.

The restrictive ADAP enrollment model based on CD4 count "served 2253 clients (37\% fewer [than had been previously served]) ... with a savings of $\$ 2.7$ million” [10]. The more restrictive policy model also resulted in " $26 \%$ savings over the... cost for an unrestricted ADAP" [10]. The unrestricted "first come, first serve approach" ADAP policy model resulted in "2406 clients served (32\% fewer [than had previously been served])" [10]. When median CD4 cell counts of both program enrollees were compared, the median cell count of those in the more restrictive policy was " 282 cells/ $\mu \mathrm{L}$," while the unrestricted policy median cell count was " 411 cells/ $\mu L$ " [10]. Thus, while the unrestricted ADAP policy served more clients, those clients tended to have higher CD4 counts.

Despite serving fewer clients, the restricted ADAP policy "decreased costs such that... the ADAP could meet the more-severe budget constraints" [10]. The restricted policy also allowed for a new and less arbitrary selection criterion based upon immediate medical need. While beyond the scope of the study, it is also possible that prioritizing treatment of those with lower CD4 counts could result in a decrease in inpatient care-related costs, as well as increase the number of lives saved due to the prevention of AIDS related morbidity. This study itself noted that "[i]f one seeks to minimize mortality, the priority should be to serve patients with low CD4 counts; these are the patients with an elevated 1-year mortality risk" [10].

Additionally, the practice of treating more vulnerable patients first is common medical practice and so does not implicate the same ethical concerns as an unrestricted approach to rationing of care. For instance, flu vaccines are first administered to the old, the sick, and the very young-those at most risk for severe viral illness. Treating those most susceptible to HIV/AIDS related illness while simultaneously adjusting the ADAP program to meet more stringent budgetary restrictions better distributes scarce resources according to urgent medical need.

Along with a standardized medical policy, state ADAP medication purchasing schemes must also be examined with regards to their budgetary impacts. States purchase HAART medications either directly from manufacturers, or from groups of retail pharmacies. While the "average costs per prescription [did] not differ between the two purchase methods," among states that purchased from retail pharmacy networks, "those that contract[ed] with smaller numbers of pharmacy networks [had] lower average costs" [8]. In attempting to reduce costs to ADAPs, one must consider whether a particular purchasing model could result in greater savings.

Along with the modification of coverage policy and the enhancement of bargaining power must come a more efficient allocation of ADAP resources. Under a new centralized ADAP program, the excess medication capacity could be re-allocated to high-need or traditionally underserved areas. This re-allocation could be directed by the federal government's Health Resources and Services Administration (HRSA). Similar to some federallydirected health insurance exchanges, a centralized ADAP administered by the HRSA would meet new national standards for beneficiary coverage. These standards would ensure greater equality across a national ADAP.

New HAART medications are particularly expensive due to patent enforcement, which prevents price competition. In developing countries, HAART medications cost "as little as $\$ 140$," while the same therapy costs "approximately $\$ 10,000$ - $\$ 15,000$ annually in the US” [8]. In the US, these costs can be mitigated by wholesale purchase of the medications. As states are high volume purchasers of HAART, they can take advantage of discounts and rebates offered by companies by "using... the rebate model or the direct purchase model" [8]. Using the rebate model, a state "negotiates with networks of pharmacies to obtain a lower price [on medications] than a retail customer without insurance would pay” [8]. Under the direct purchase model, a state uses its own central pharmacy to purchase the medications directly from the manufacturer [8].

In one study using multivariate analysis, "regressions [were conducted] that related... spending for a given state... to the type of [HAART medication] and the ADAP model used by the state in the year" [8]. The results indicated that states which negotiated directly with manufacturers for ADAP medications did not receive lower prices than states which used rebate purchasing models. However, further analysis of state rebate models indicated that in states with larger pharmacy networks, ADAP medications "had higher costs per prescription than [in] rebate states that use[d] fewer pharmacies" [8]. Therefore, a more efficient medication rebate procurement model might require buying from a consolidated or single pharmacy network.

States are able to receive lower medication prices by leveraging the threat of "reducing drug purchases from a given manufacturer" [8]. This only constitutes a marginal advantage, because states must act according to their own separate and distinct purchasing models. As these models vary by state, drug manufacturers possess the advantage of being able to negotiate with separate medication procurement offices. For this reason, if all states ceded their purchasing authority to a single national/federal procurement entity, "[they] might [attain] the lowest average prices" [8]. However, having every state adopt a national ADAP purchasing authority would require a national ADAP policy standard. 


\section{ADAP and the Passage of Healthcare Reform}

A national ADAP standard would benefit program enrollees and reduce costs. The standard would allow for the creation of a single ADAP medical enrollment criterion and federal medication procurement office. This centralization would better address budgetary issues as well as issues related to program equity and sustainability. Under this new national policy, every state with an ADAP would be required to surrender its primary programmatic authority to the federal government. Although achieving this degree of compliance would certainly be an arduous political undertaking, as "part of a new omnibus healthcare reform act, a national ADAP policy might be feasible” (B. Miller, personal communication, April 2, 2012). While no such national ADAP policy initiative currently exists, future efforts related to healthcare reform might benefit from the long-term cost savings associated with a national HIV/AIDS drug program. Future cost-benefit analyses should be conducted in this area. In contemplating the feasibility of a national ADAP standard, one should first understand the political difficulty of implementing healthcare reform, a similar health standard initiative, in the United States.

Health care reform remains one of the most complex and politically divisive issues in America today. At the center of the debate are budgetary and political issues with an essential division existing between two differing ideologies. Both ideologies advance very different views on what the role of the federal government should be in relation to the welfare of its citizens. Generally, political progressives, supported by the Democratic party want to make quality health care available to all citizens, while political conservatives, supported by the Republican party are concerned about having a government which exceeds its mandate. At the center of the debate are budgetary issues-primarily, how health care will be paid for in the future.

Despite being unpopular to many Americans in its entirety, healthcare reform, namely the Patient Protection and Affordable Care Act, contains many popular provisions. Among these provisions are the expansion of health insurance coverage to include more low-income individuals under Medicaid, as well as new laws which prevent the practice of rescission (L. Nichols, personal communication, November 15, 2011). Additionally, the ACA's policies on creation of 'insurances exchanges' for small businesses, allowing children to stay on their parents insurance until the age of twenty-six, and prohibiting insurance companies from discriminating against consumers because of a pre-existing condition receive overwhelming support from both parties when considered in isolation [11]. It is also worth noting that while the majority of surveyed Americans would want to "repeal the health care legislation," the majority of surveyed Americans also believe that "the current health care system is [subpar]" [12]. In light of these facts, the political hurdles that might face the enactment of a national ADAP might be overcome if its individual components can be "sold" to the public in the right way. If the passage of the ACA provides any guidance in this area, it is most likely to be that sweeping change to existing policy is sometimes better sold to the public in pieces rather than the largescale reform measure that it is.

Experts remain uncertain as to whether proactive health care reform will save costs into the future. While those with chronic health conditions such as HIV/AIDS, the previously uninsured, and individuals with lowincome would immediately benefit from the reform, the "increased administrative costs associated with reform might continue to weigh on economic growth in the long run” (B. Hoagland, personal communication, November 17, 2011). As with healthcare reform, experts also remain uncertain as to the long-term viability of ADAP [4]. However, these skeptics must also consider the potential cost savings associated with a national program.

Without program reform or drastic budgetary reductions, ADAP, like "the current (unreformed) healthcare system, is not sustainable into the future” (B. Miller, personal communication, April 2, 2012). Many cost studies on ADAPs suggest that creating a more efficient policy requires centralization. This centralization might be best achieved under the umbrella of healthcare reform and the creation of a national healthcare standard. This standard might allow for the development of more efficient ADAP medical and procurement policy. A new national ADAP policy might also keep the program at pace with the complexities associated with long-term HIV/AIDS care. Otherwise, short of finding a cure for the HIV virus, all that remains to be done is to further ration already diminished resources at the state level.

The consolidation of ADAP procurement only addresses the issue of cost. Medical resource disparities between states, namely their medication distribution systems and health administration infrastructure must also be examined. While beyond the immediate scope of this paper, policies aimed at reducing state health system inequalities must also be considered in tandem with the implementation of a new ADAP policy. Without the prerequisite systematic reform of the overall US health care system, the results of a new national ADAP will only reflect the efficiency and viability of individual state health care systems.

\section{Conclusions}

Despite its immediate effectiveness, ADAP in its current 
form is in danger of budgetary constrainting largely the result of the economic recession. Current state-managed ADAPs also creates disparities in coverage: to the patient population generally based on unrestricted 'first come, first served' policies, and to minorities in particular. The cost of HAART treatment is ever higher, and, as it continues to increase the life expectancy of current HIV/ AIDS patients, which will continue to increase the cost of therapy over a given individual's lifetime.

States alone can decrease costs. This can be done firstly through programmatic changes that base coverage on medical need instead of a patient's place in line, and secondly by employing different bargaining methods for drugs. However, since an individual state must do this alone, the overall savings to a given state-managed ADAP are limited. Thus, the most effective method is a national uniform policy that allows for centralized administration of coverage policies and larger bargaining power for costly HAART drugs. Certainly, such a proposal would face political hurdles, but the recent passage and enactment of healthcare reform through the ACA could provide guidance as to how to overcome those hurdles. Ultimately, a national policy would lead to more efficient practices within ADAP and increase its long-term sustainability.

\section{REFERENCES}

[1] US Department of Health and Human Services, "Going the Distance: The Ryan White HIV/AIDS Program. 20 Years of Leadership, A Legacy of Care,” 2010. http://www.impactmc.net/downloads/samples/writing/Goi ng-the-Distance-20-years-of-the-Ryan-White-HIV-AIDSProgram.pdf

[2] M. Johri, D. A. Paltiel, S. J. Goldie and K. A. Freedberg, "State AIDS Drug Assistance Programs: Equity and Efficiency in an Era of Rapidly Changing Treatment Standards,” Medical Care, Vol. 40, No. 5, 2002, pp. 429-441.

[3] D. P. Goldman, J. Bhattacharya, A. A. Leibowitz, G. F.
Joyce, M. F. Shapiro and S. A. Bozzette, "The Impact of State Policy on the Costs of HIV Infection," Medical Care Research and Review, Vol. 58, No. 1, 2001, pp. 31-53.

[4] B. R. Schackman, K. A. Gebo, R. P. Walensky, E. Losina, T. Muccio, P. E. Sax, et al., "The Lifetime Cost of Current Human Immunodeficiency Virus Care in the United States," Medical Care, Vol. 44, No. 11, 2006, pp. 990997. doi:10.1097/01.mlr.0000228021.89490.2a

[5] Kaiser Family Foundation, "2009 National ADAP Monitoring Project Annual Report,” The Henry J. Kaiser Family Foundation, 2009.

http://kff.org/hivaids/event/2009-national-adap-monitorin g-project-annual-report/

[6] R. P. Walensky, A. D. Paltiel and K. A. Freedman. “AIDS Drug Assistance Programs: Highlighting Inequities in Human Immunodeficiency Virus-Infection Health Care in the United States," Clinical Infectious Diseases, Vol. 35, No. 5, 2002, pp. 606-610. doi:10.1086/341903

[7] S. Morin, "Responding to Racial and Ethnic Disparities in Use of HIV Drugs: Analysis of State Policies," Public Health Reports, Vol. 117, No, 3, 2002, pp. 263-272.

[8] A. A. Leibowitz and N. Sood, "Market Power and State Costs of HIV/AIDS Drugs," International Journal of Health Care Finance and Economics, Vol. 7, No. 1, 2007, pp. 59-71. doi:10.1007/s10754-007-9012-0

[9] J. Kahn, “Access to and Use of HIV Antiretroviral Therapy: Variation by Race/ethnicity in Two Public Insurance Programs in the US,” Public Health Reports, Vol. 117, No. 3, 2002, pp. 252-262.

[10] B. P. Linas, H. Zheng, E. Losina, A. Rockwell, R. P. Walensky, K. Cranston, et al., "Optimizing Resource Allocation in United States AIDS Drug Assistance Programs,” Clinical Infectious Diseases, Vol. 43, No. 10, 2006, pp. 1357-1364. doi:10.1086/508657

[11] J. McCarter, “Obamacare Unpopular, Unless It’s Not Called Obamacare,” Daily Kos, 2012.

[12] Pew Research Center, "Health Care Proposals Remain Hard to Follow,” Pew Research Center for the People and the Press, 2009.

http://www.people-press.org/2009/09/08/health-care-prop osals-remain-hard-to-follow/ 